$$
\text { التآكل الجوي للصلب الكربوني وحديد الزهر الرمادي في بيئة جنوب الموصل }
$$

$$
\text { مدرس مساعد إلمعهد التقتي/ألاور }
$$

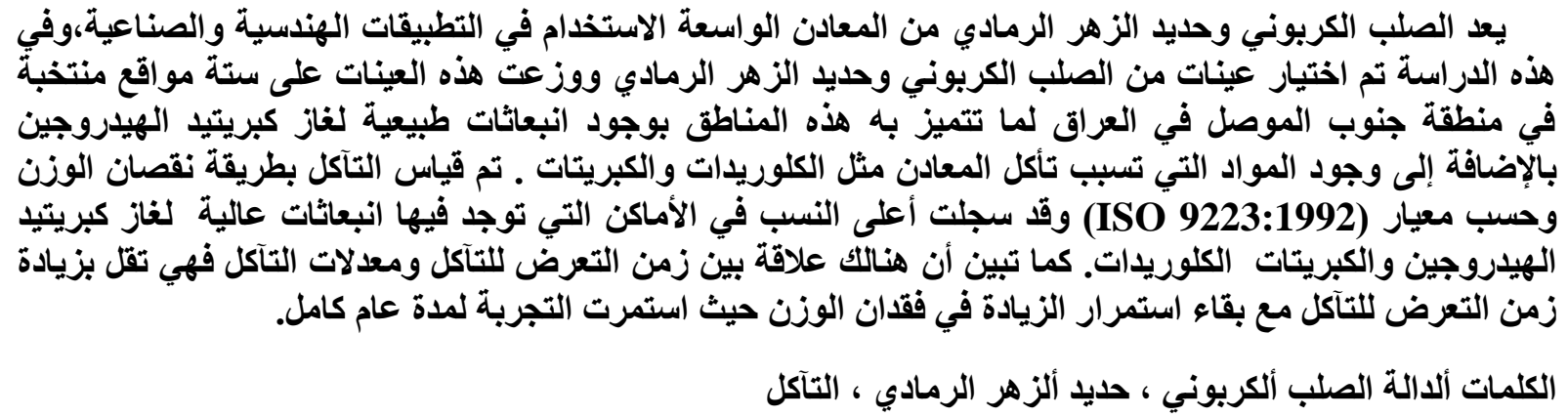

\title{
The Atmospheric Corrosion of Carbon Steel and Cast Iron in the Environment of South Mosul
}

\section{Nadum Ibrahim Naser}

\author{
Assistant lecturer Technical Institute in dour
}

\begin{abstract}
The carbon steel and cast iron are widely used metals in engineering and industrial applications. In this study, samples of carbon steel and cast iron chosen and distributed on six selected sites in the south region of Mosul city in Iraq, Because these sites distinguished by the existence of natural emanations of hydrogen sulfide gas is produced and the existence of materials that cause metal corrosion like sulfates and chlorides. The corrosion measured by weight loss method according to the standard (ISO 9223:1992) and the higher rates registered in the sites that contain high emanations of hydrogen sulfide gas and sulfates and chlorides. In addition, it became obvious that there is a relationship between the corrosion exposure time and the corrosion rates which decreased by increasing of corrosion exposure time and continuous increasing in weight loss. The test continued for a complete year.
\end{abstract}

Key words: carbon steel, cast iron, corrosion. 


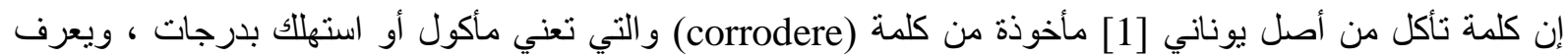

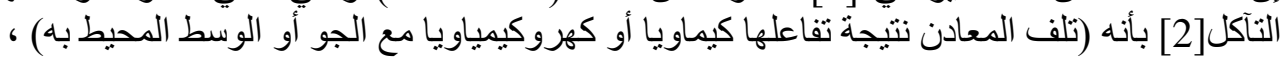

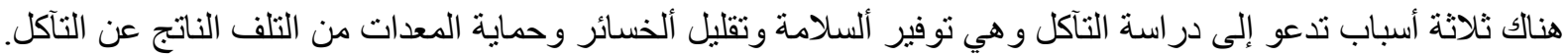

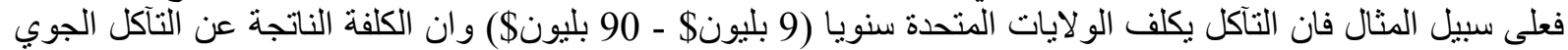

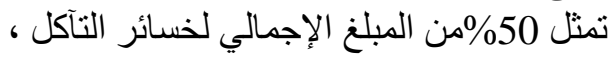

يعتبر التآكل الجوي عملية كهروكيميائية معقدة تؤدي إلى تكوين خلايا التآكل و التي تسبب انحلال المعدن[3] . إن التآكل له علاقة وثثقة بنو عية المادة و الوسط ألمساعد وان من أهم الأوساط المساعدة هو المحيط الجوي و الماء وطبيعة التربة.

إن المحيط الجوي هو أكثر و أسر ع الأوساط المساعدة على التآكل انتشار ا وشيو عا حيث أن تأثيره في عملية التآكل لا يمكن إز الته كما هو في الأوساط الأخرى.

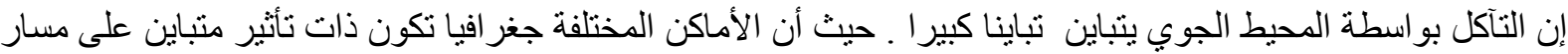
التآكل وسر عته بالإضافة إلى نأثير المحيط الجوي الخارجي منل أوساط المناطق الريفية الرية والمين المدن و المناطق الساحلية.

إن أهم مركبات المحيط الجوي الطبيعي هو النيتروجين والأوكسجين وبخار الماء إضافة إلى مركبات أخرى يعتمد تو اجدها

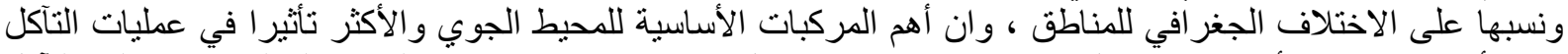

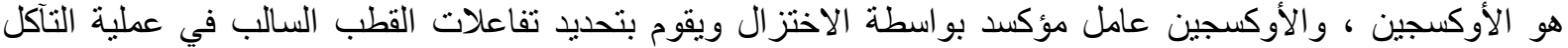
الاليكتروليتي وكما في المعادلات التالية [4] فئنين في المحاليل الحمضية

$\mathrm{O}_{2}+4 \mathrm{H}^{+}+4 \mathrm{e}^{-}=2 \mathrm{H}_{2} \mathrm{O}$

$\mathrm{O}_{2}+2 \mathrm{H}_{2} \mathrm{O}+4 \mathrm{e}^{-}=4 \mathrm{OH}^{-}$

في المحاليل القاعدية

ولأجل حصول هذا التفاعل الاليكتروليتي. الاليكتروليت (محلول موصل للكهرباء) حيث أن بخار الماء الموجود في المحيط

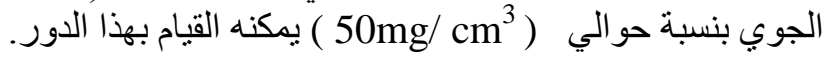

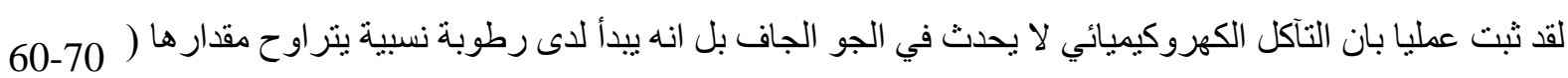
(mg/cm³ قطر ات من السائل تكفي لحدوث التآكل .

يعتبر الحديد من المعادن التي لا نتواجد في الطبيعة كمعدن نقي ولكن نوجد على شلى شكل مركبات كيميائية مثل الاكاسيد

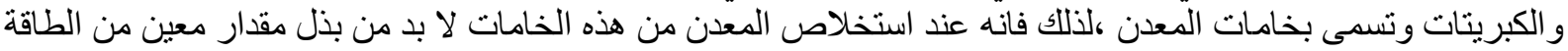

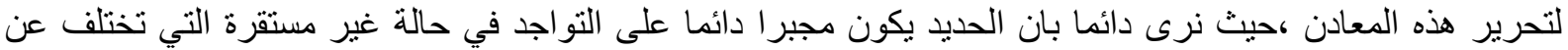

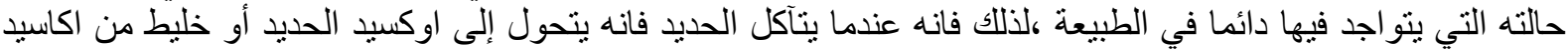

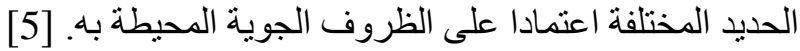

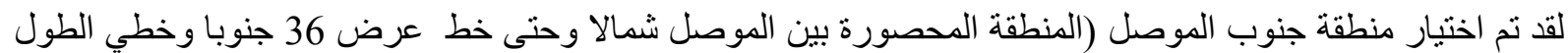

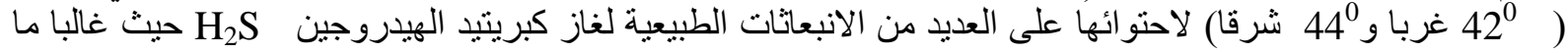




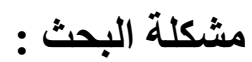

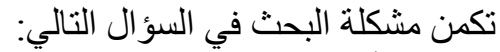
ما مدى تأثثر الانبعاثات الجوية الطبيعية لمنطقة جنوب الموصل في درجة التآكل لحديد الزهر الرمادي و الصلب الكربوني.

\section{هدف البحث:}

يهرف البحث الى التعرف على مدى تأثير العوامل الجوية في تآكل حديد الزهر الرمادي والصلب الكربوني في منطقة

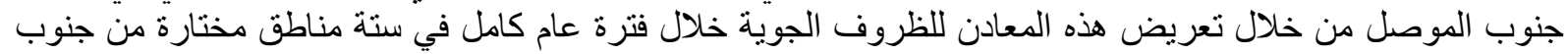

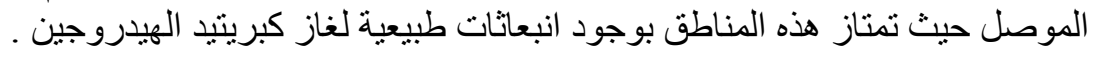

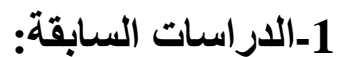

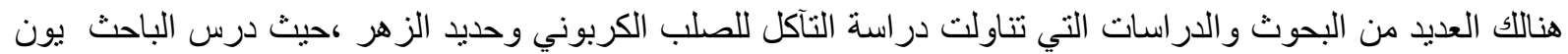

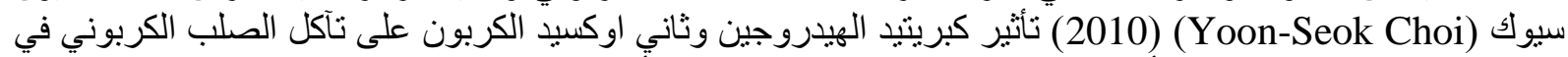

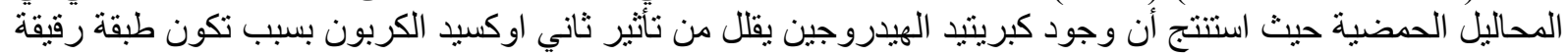

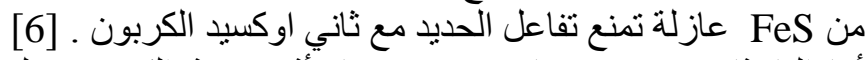

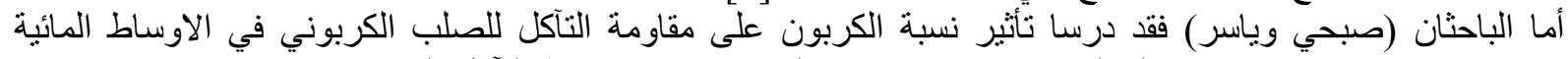

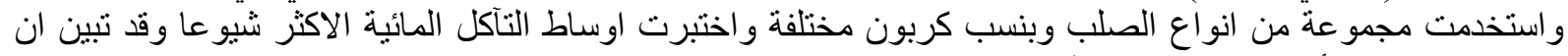

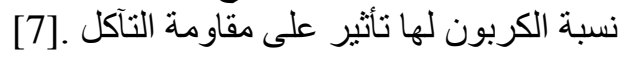

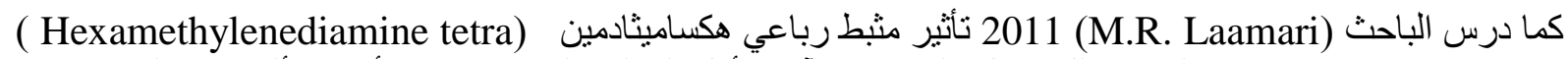
حيث اكتثف بان وجود هذا المثبط يقلل بشكل ملحوظ من آثار تأكل الصلب الكربوني في الكئل الأوساط ألحمضية لتكون طبقة

رقيقة تحيط بالصلب الكربوني تمنع استمر ار التآكل. [8.

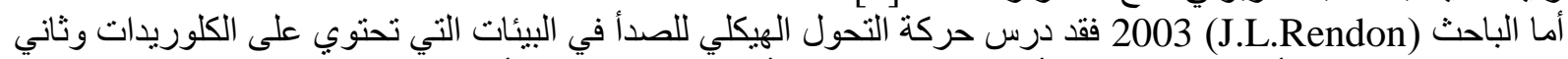

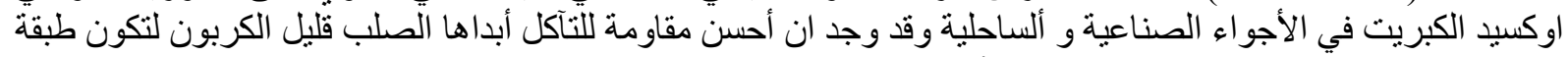

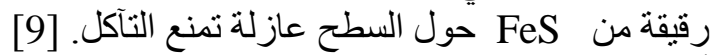

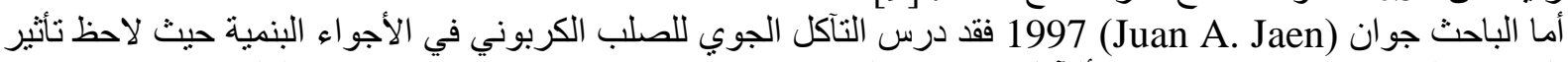

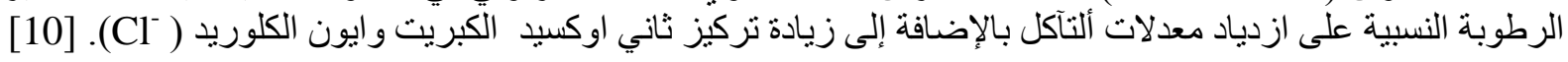

\section{2- سلوك الصلب الكربوني وحديد الزهر الرمادي تحت ظروف التآكل:}

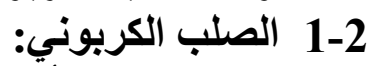

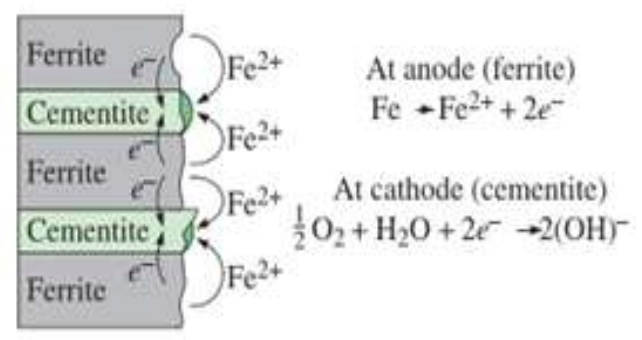

شكل (1) التآكل الجوي للصلب الكربوني

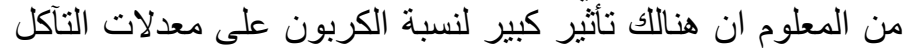

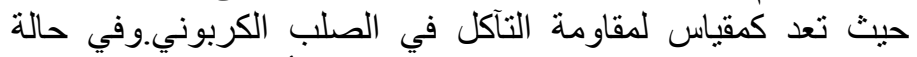

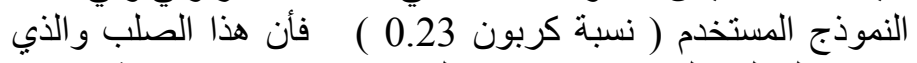

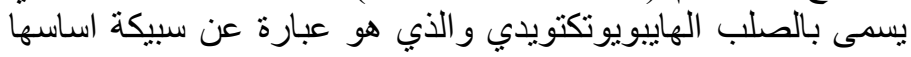

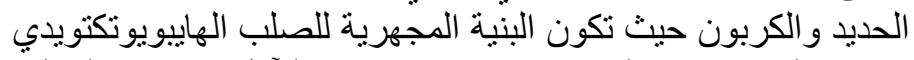

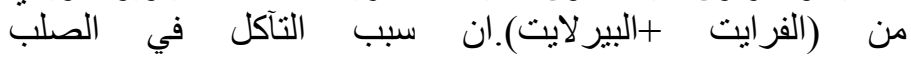

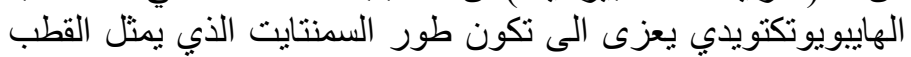

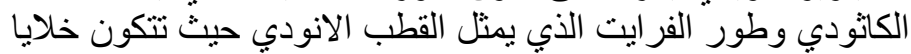
كلفانية منمنلة بالبيلايت الذي يتكون من التئ (الفرايت +السمنتايت)

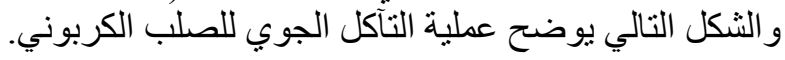


اما في حالة حديد الزهر الرمادي (نسبة الكربون 3.53) فان سبب التآكل ناتج ايضا عن تكون الخلايا الكلفانية حيث ان ان الكئ

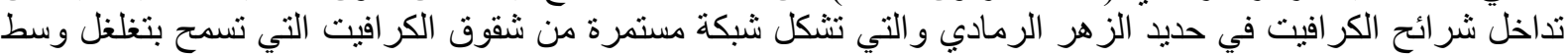

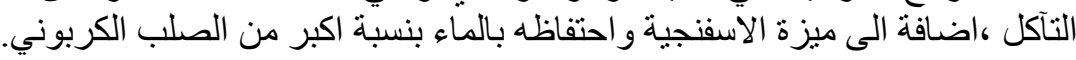
والثكل يوضح عملية التآكل الجوي لحديد الزهر الرمادي[11] :

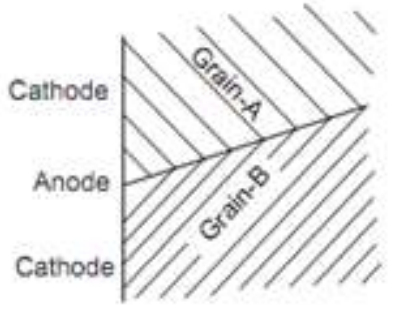

(a)

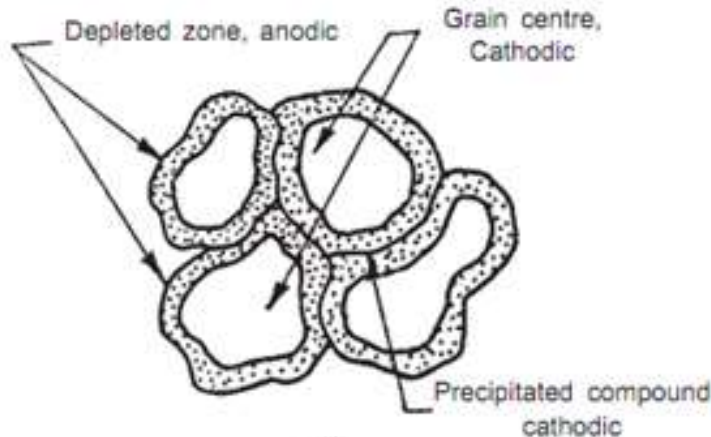

(b) cathodic

شكل (2) التآكل الجوي لحديد الزهر الرمادي

$2 \mathrm{Fe} \rightarrow 2 \mathrm{Fe}^{2-}+4 e^{-}$

$\mathrm{O}_{2+}+2 \mathrm{H}_{2} \mathrm{O}+4 \mathrm{e}^{-} \rightarrow 4 \mathrm{OH}^{-}$

( cathodic reaction)

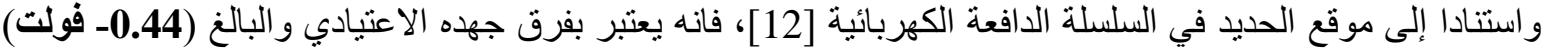

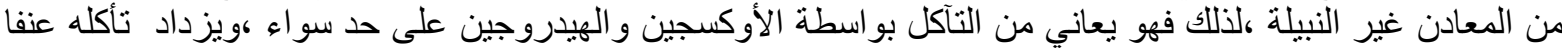

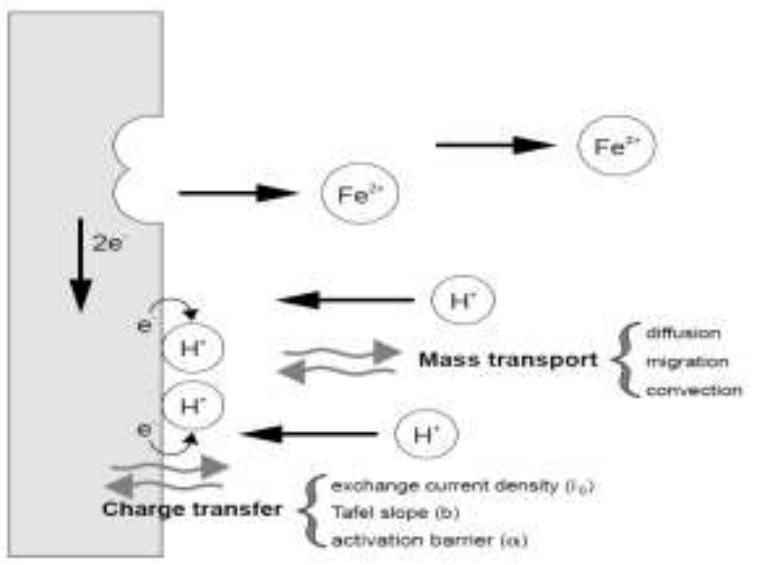

شكل (3) بداية تكون السلبية (passivity) في الحديد

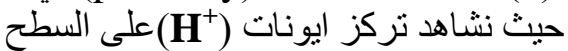

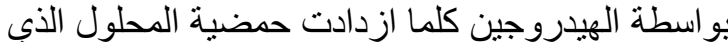

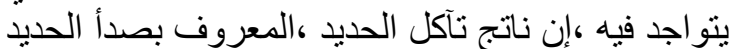

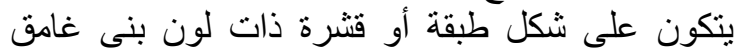

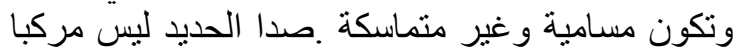
كيماويا متجانسا ، بل انه ينكون من خليط من الاكاسيد

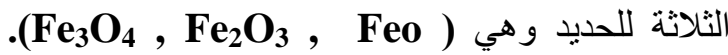
يعاني الحديد من الحوامض الإوكسيدية الحاوية على الحئ

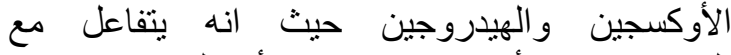

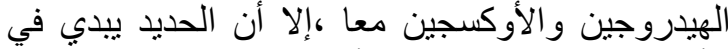

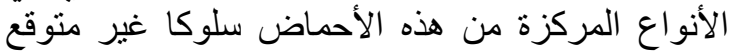

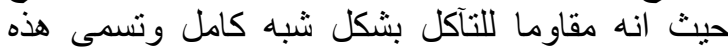

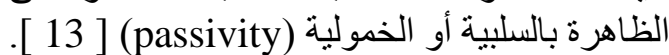

يزداد تأكل الحديد في المحبط الجوي وخاصة بازدياد

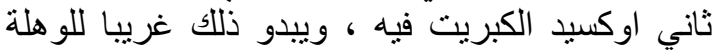
الأولى ،حيث أن هذا الاوكسيد عامل مختزل ،إلا أن مفعول هذا الاوكسيد في زيادة نتآكل الحديد لا ينتج من الاوكسيد نفسه [14]: بل من حامض الكبريتيك الذي يتكون بموجب التفاعل 
$\mathrm{Fe}+\mathrm{SO}_{2}+\mathrm{O}_{2}=\mathrm{FeSO}_{4}$

$4 \mathrm{FeSO}_{4}+\mathrm{O}_{2}+6 \mathrm{H}_{2} \mathrm{O}=2 \mathrm{Fe}_{2} \mathrm{O}_{3}+\mathrm{H}_{2} \mathrm{O}+4 \mathrm{H}_{2} \mathrm{SO}_{4}$

$2 \mathrm{Fe}+2 \mathrm{H}_{2} \mathrm{SO}_{4}+\mathrm{O}_{2}=2 \mathrm{FeSO}_{4}+2 \mathrm{H}_{2} \mathrm{O}$

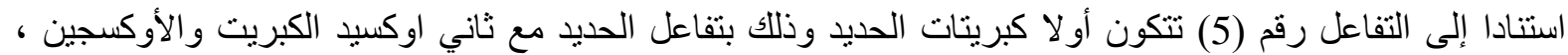

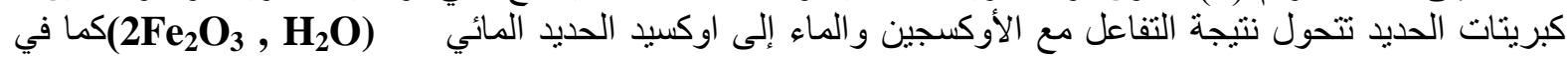

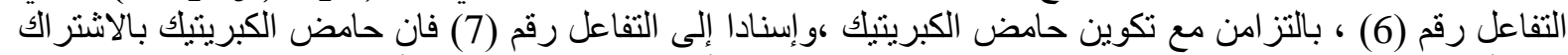

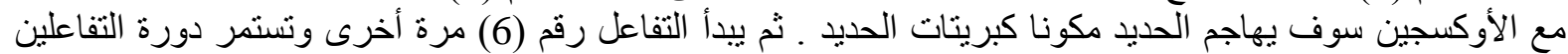

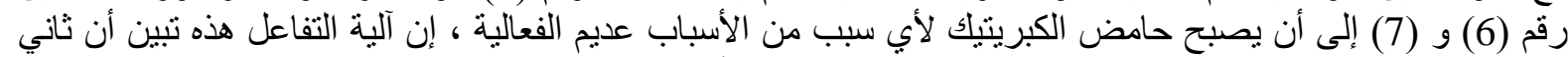

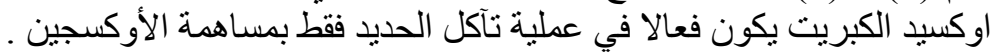

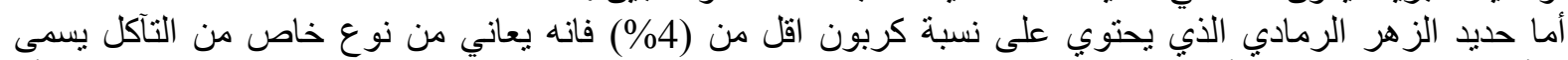

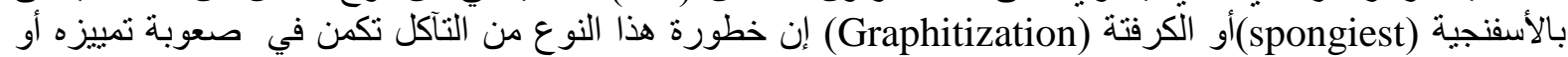

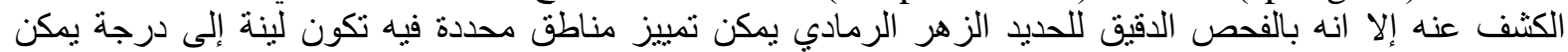

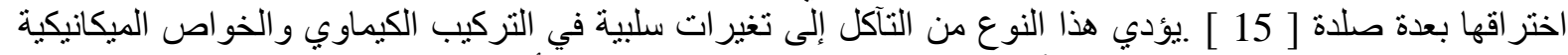
للحديد الزهر إن سبب هذا النوع من التآكل هو إذابة الحديد النقي تحت تكوين أول اوكسيد الحديد (FeO)في حين يبقى الكربون على شكل كر افيت لينا.

\section{3- خصائص منطقة جنوب الموصل}

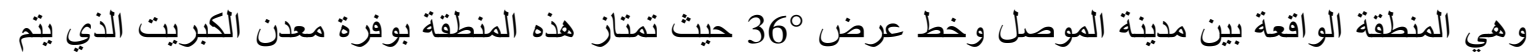

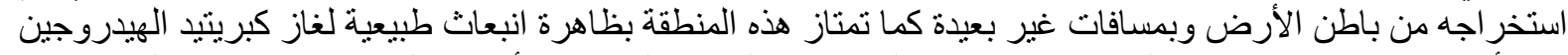

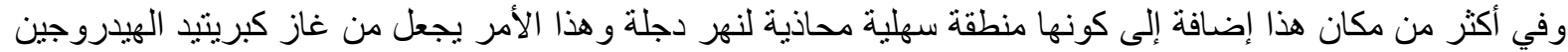

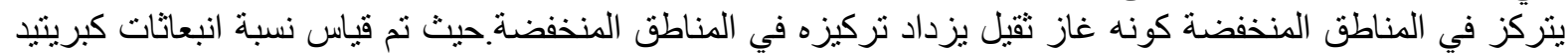

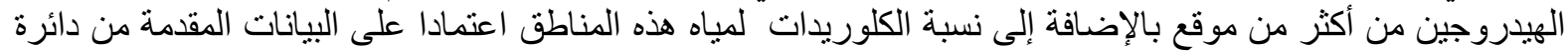

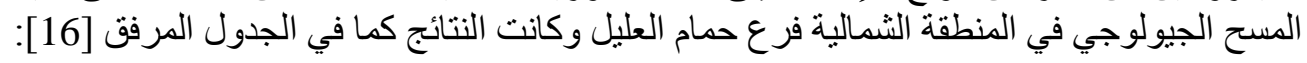

جدول (1) النسب المئوية للعناصر المسببة للتآكل

\begin{tabular}{|c|c|c|c|c|c|}
\hline $\mathrm{CO}_{2} \mathrm{Mg} / \mathrm{L}$ & $\mathrm{SO}_{2} \mathrm{Mg} / \mathrm{L}$ & Cl Mg/L & $\mathrm{H}_{2} \mathrm{~S} \mathrm{Mg} / \mathrm{L}$ & المكان & $ت$ \\
\hline 425 & 1263.5 & 3105.4 & 648.2 & المشر اق(1) & 1 \\
\hline 304 & 1600 & 1594.3 & 414.6 & المشر اق(2) & 2 \\
\hline 90.9 & 26.3 & 168.5 & 63.2 & حمام العليل & 3 \\
\hline 108 & 150.6 & 207 & 111.3 & تل الهشم & 4 \\
\hline 68.5 & 1667.2 & 842.2 & 73.3 & عين البيضـا & 5 \\
\hline 20.9 & 1960.6 & 141.8 & 161.3 & المستتطق & 6 \\
\hline
\end{tabular}

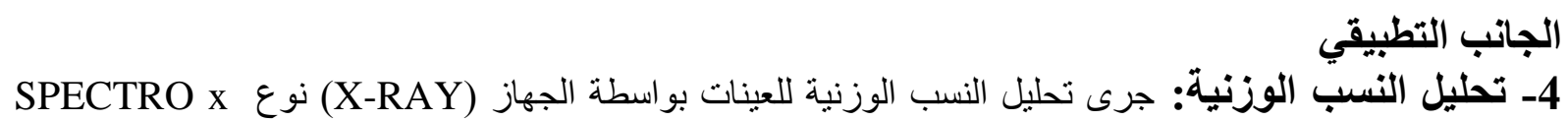

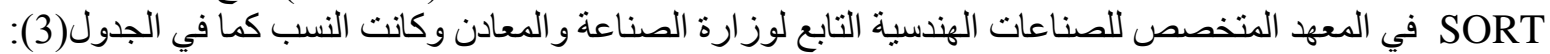

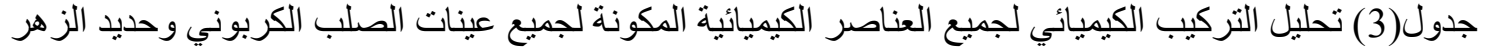

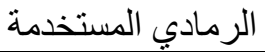

\begin{tabular}{|c|c|c|c|c|c|c|c|c|c|}
\hline \multirow[t]{2}{*}{ النماذج } & \multicolumn{9}{|c|}{ النسب الوزنية \% لعينات الصلب الكربوني وحديد الزهر الرمادي } \\
\hline & $\mathrm{C}$ & $\mathrm{Si}$ & $\mathrm{Mn}$ & $\mathrm{P}$ & S & $\mathrm{Cr}$ & $\mathrm{Ni}$ & $\mathrm{Al}$ & $\mathrm{Fe}$ \\
\hline الصلب الكربوني & 0.23 & 0.39 & 0.4 & 0.08 & 0.05 & 0.1 & 0.43 & 0.04 & Balance \\
\hline حديد الز هر الرمادي & 3.53 & 2.8 & 1.3 & 0.04 & 0.25 & 0.23 & 0.03 & 0.02 & Balance \\
\hline
\end{tabular}




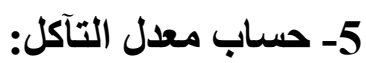

من المعروف بان معدل اختراق التآكل هو في الغالب دالة غير خطية بالنسبة للزمن وان تأكل المبل المعدن بقل بمرور الزمن

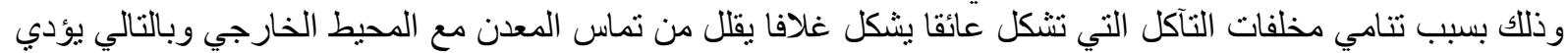

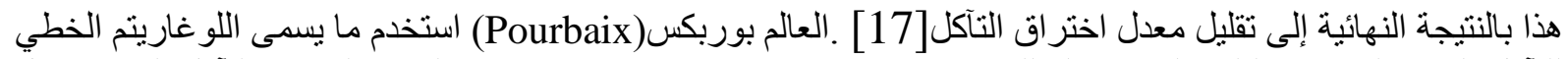

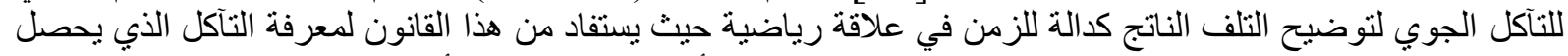

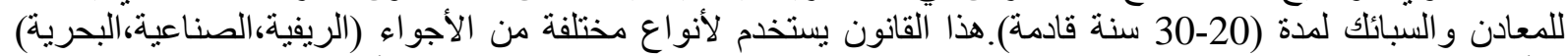

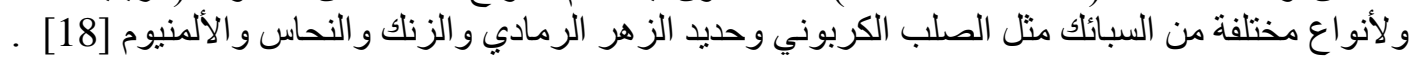

$M=a T^{b} \ldots \ldots \ldots \ldots$ حيث:

$\mathrm{M}=($ Corrosion Rate $)$

$\log M=a^{*}+b \log T$ $a^{*}-\log a$

حيث تمثل:

CR Ammy 'معدل اختراق التآكل بالنسبة لوحدة المساحة = M

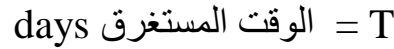
= خسارة الوزن للسنة الأولى a

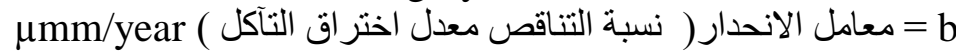
بأخذ لو غاريتم الطرفين فتصبح المعادلة:

هذه المعادلة تستخدم لإيجاد مقدار اختر اق ألتآكل لأكثر من سنة عن طريق معادلة خطية .

1-5 إيجاد الفقدان بالوزن (Weight Loss Measurements):

) تم إعداد النماذج (10mm×15mm×3mm) في الثكل والحجم المطلوبين وفق معيار

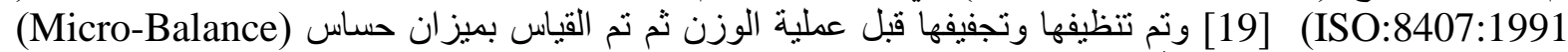

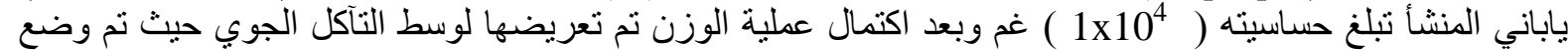

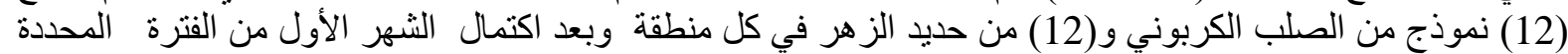

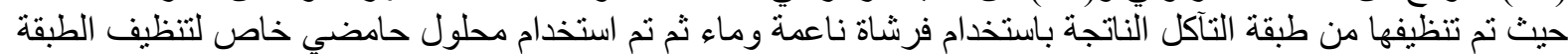

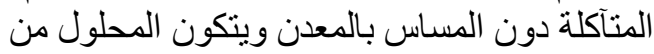

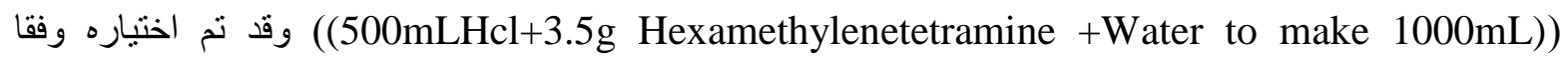

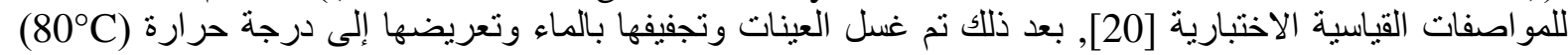

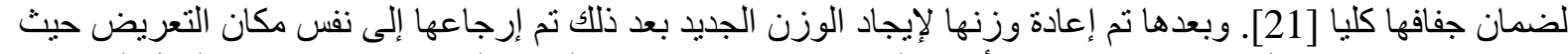

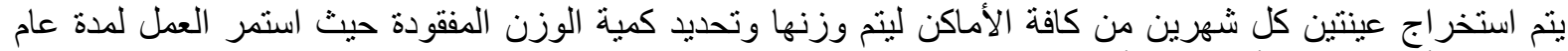

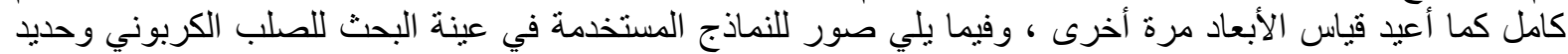
الزهر قبل وبعد التآكل: 
ناصر: التآكل الجوي للصلب الكربوني وحديد الزهر الرمادي في بيئة جنوب الموصل

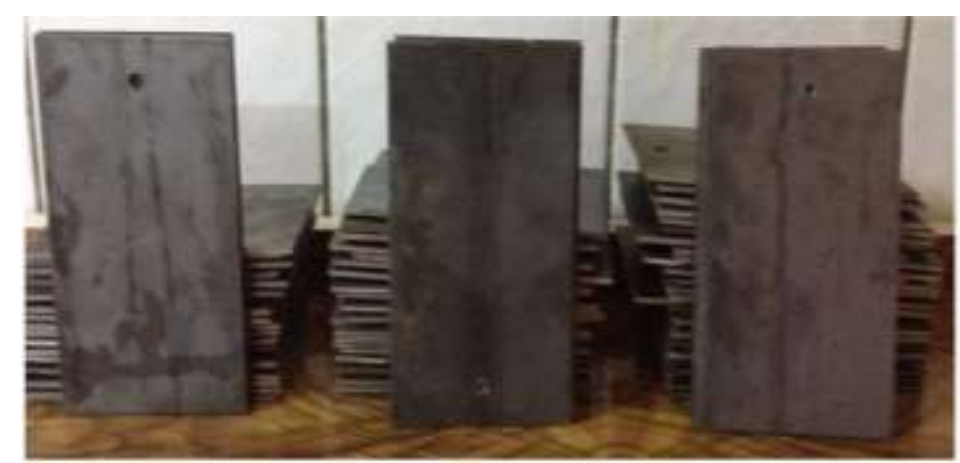

صورة (1) عبنات الصلب الكربوني قبل التآكل
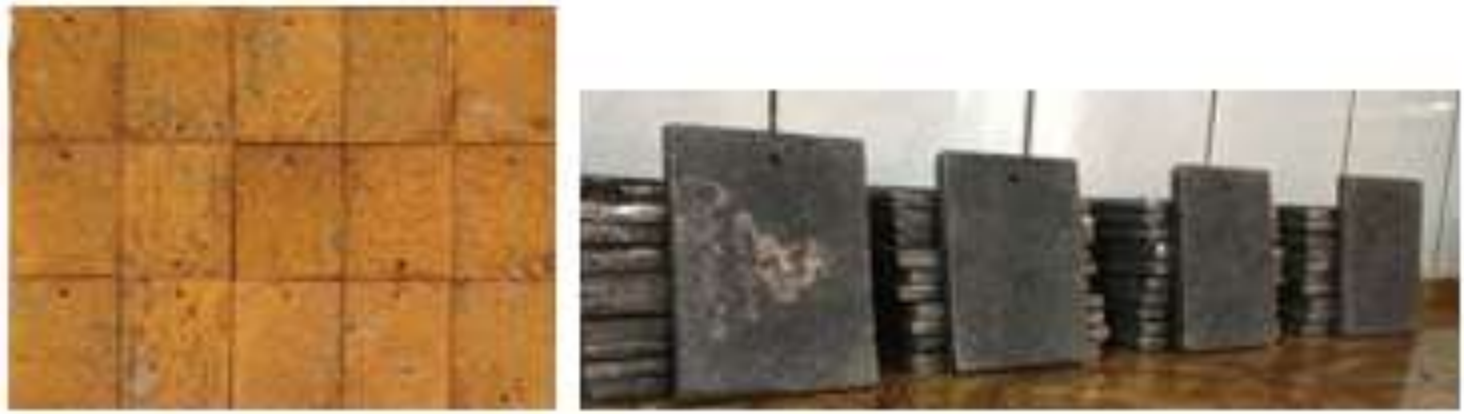

صورة (2) عينات الصلب الكربوني بعد التآكل

صورة (3) عينات حديد الزهر قبل التآكل

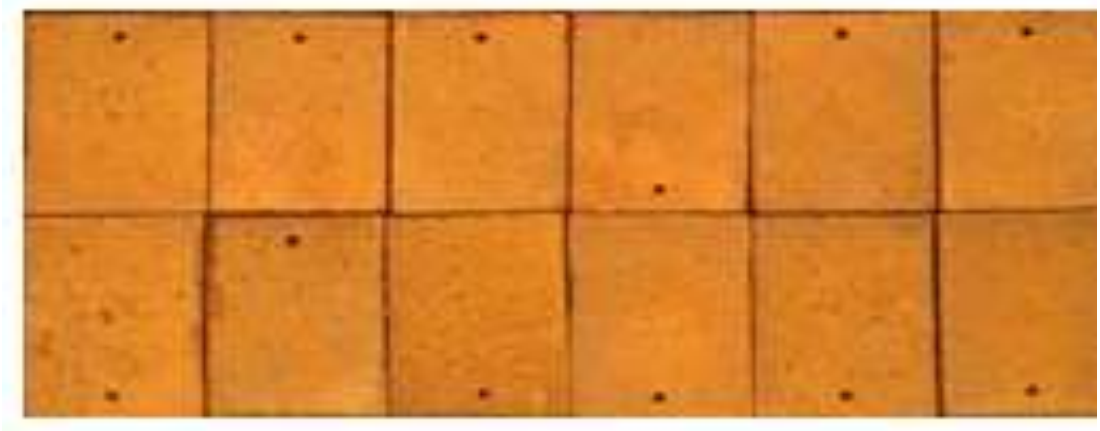

صورة (4) عينات حديد الزهر بعد التآكل

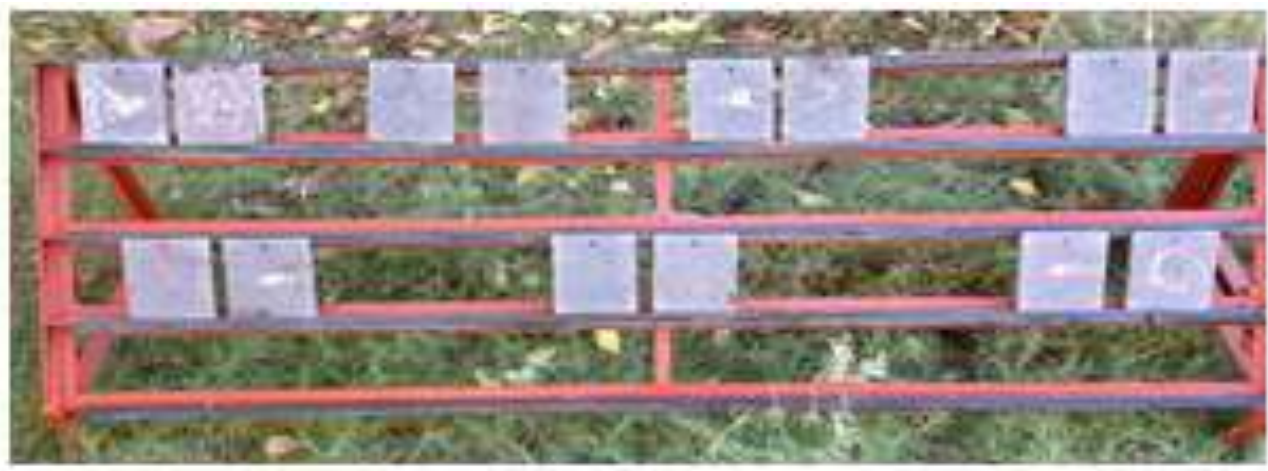

$$
\text { صورة (5) وضع عينات حديد الزهر في احد الحقول }
$$




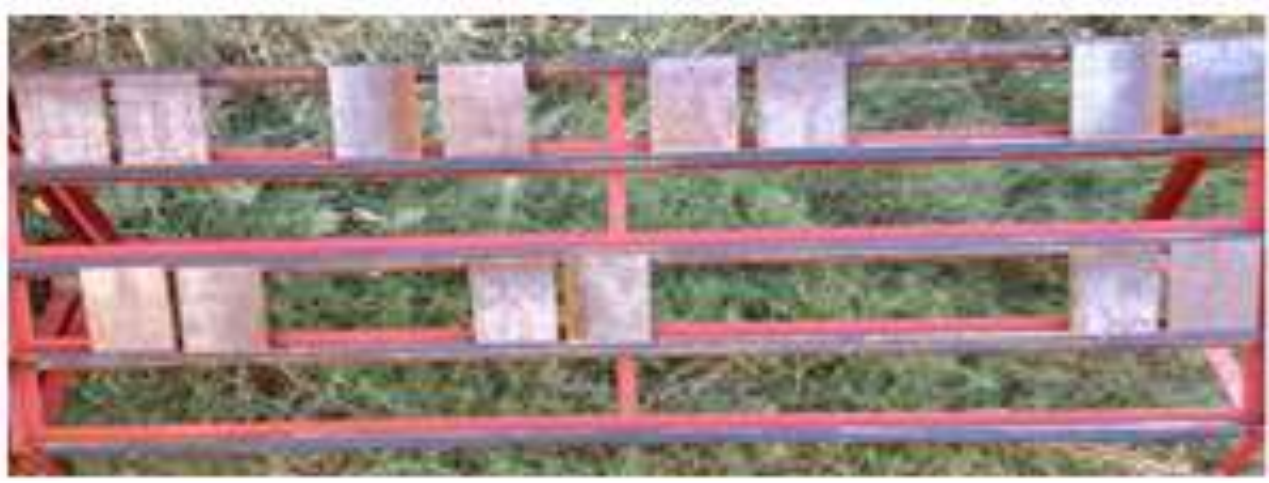

$$
\text { صورة (6) وضع عينات الصلب الكربوني في احد الحقول }
$$

\section{6- النتائج والمناقشة:}

كانت كمية الخسارة في الوزن كما في الجدول المرفق.

جدول (2) كمية الخسارة الوزنية بالغر امات لمدة عام واحد

\begin{tabular}{|c|c|c|c|c|c|c|c|}
\hline الكلية للوزارن & التاسعر & الشابع & الخامسر & الثالثر & الأول & المنطقة & النوع \\
\hline 2.176 & 1.92 & 1.73 & 1.42 & 0.87 & 0.56 & المشراق (1) & \multirow{6}{*}{ 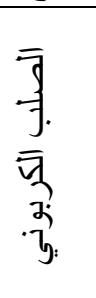 } \\
\hline 1.909 & 1.57 & 1.39 & 1.09 & 0.76 & 0.55 & المشر اث (2) & \\
\hline 1.439 & 1.09 & 0.84 & 0.73 & 0.52 & 0.44 & حمام العليل & \\
\hline 2.174 & 1.87 & 1.65 & 1.22 & 0.74 & 0.53 & تل الهشم & \\
\hline 1.977 & 1.61 & 1.33 & 0.94 & 0.65 & 0.42 & عين البيضسا & \\
\hline 1.975 & 1.67 & 1.35 & 0.97 & 0.78 & 0.45 & المستنطق & \\
\hline 2.725 & 2.47 & 1.94 & 1.48 & 0.95 & 0.55 & المشر اق (1) & \multirow{6}{*}{$\begin{array}{l}\frac{1}{3} \\
\overline{3} \\
3 \\
\overline{3} \\
\overline{3} \\
\overline{3}\end{array}$} \\
\hline 2.448 & 2.25 & 1.98 & 1.42 & 0.91 & 0.54 & المشر اق (2) & \\
\hline 1.812 & 1.60 & 1.49 & 1.19 & 0.75 & 0.43 & حمام العليل & \\
\hline 2.506 & 2.13 & 1.86 & 1.32 & 0.96 & 0.52 & تل الهشم & \\
\hline 2.015 & 1.88 & 1.56 & 1.19 & 0.79 & 0.43 & عين البيضـا & \\
\hline 2.1038 & 1.82 & 1.53 & 1.11 & 0.78 & 0.45 & المستتطق & \\
\hline
\end{tabular}

و المخطط التالي يوضح كمية الخسارة الوزنية بالغر امات لمدة عام كامل

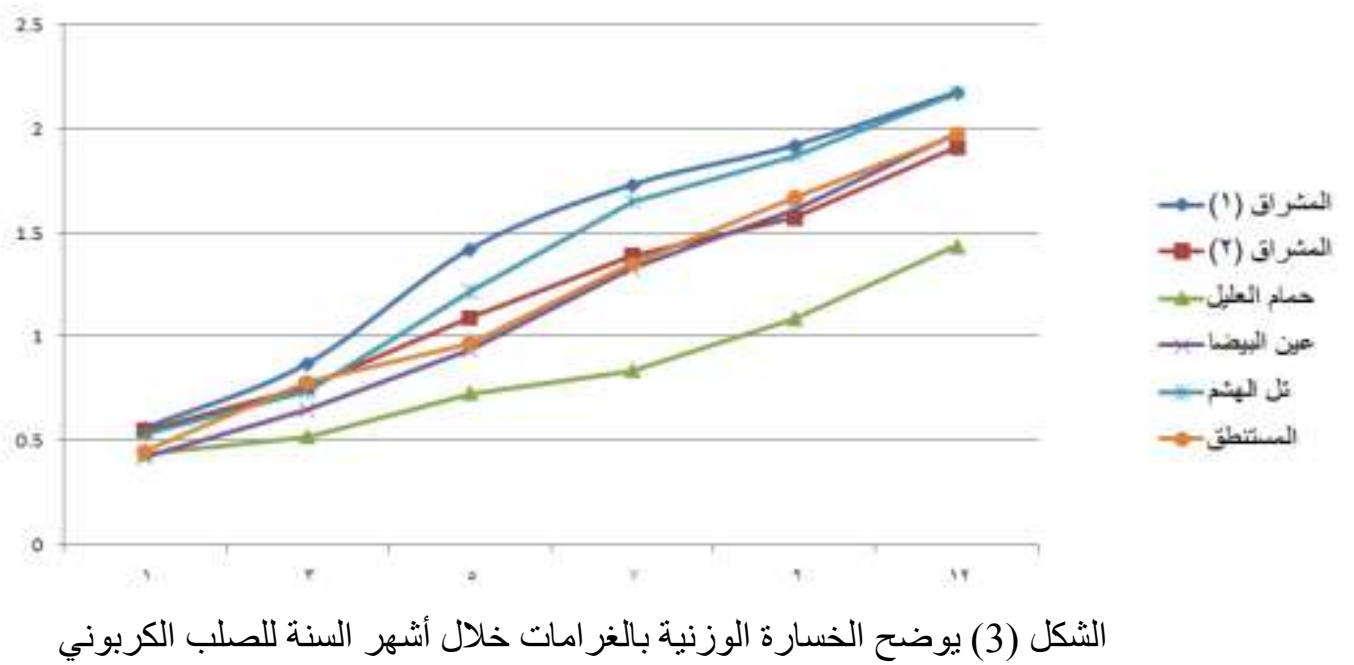


ناصر: التآكل الجوي للصلب الكربوني وحديد الزهر الرمادي في بيئة جنوب الموصل

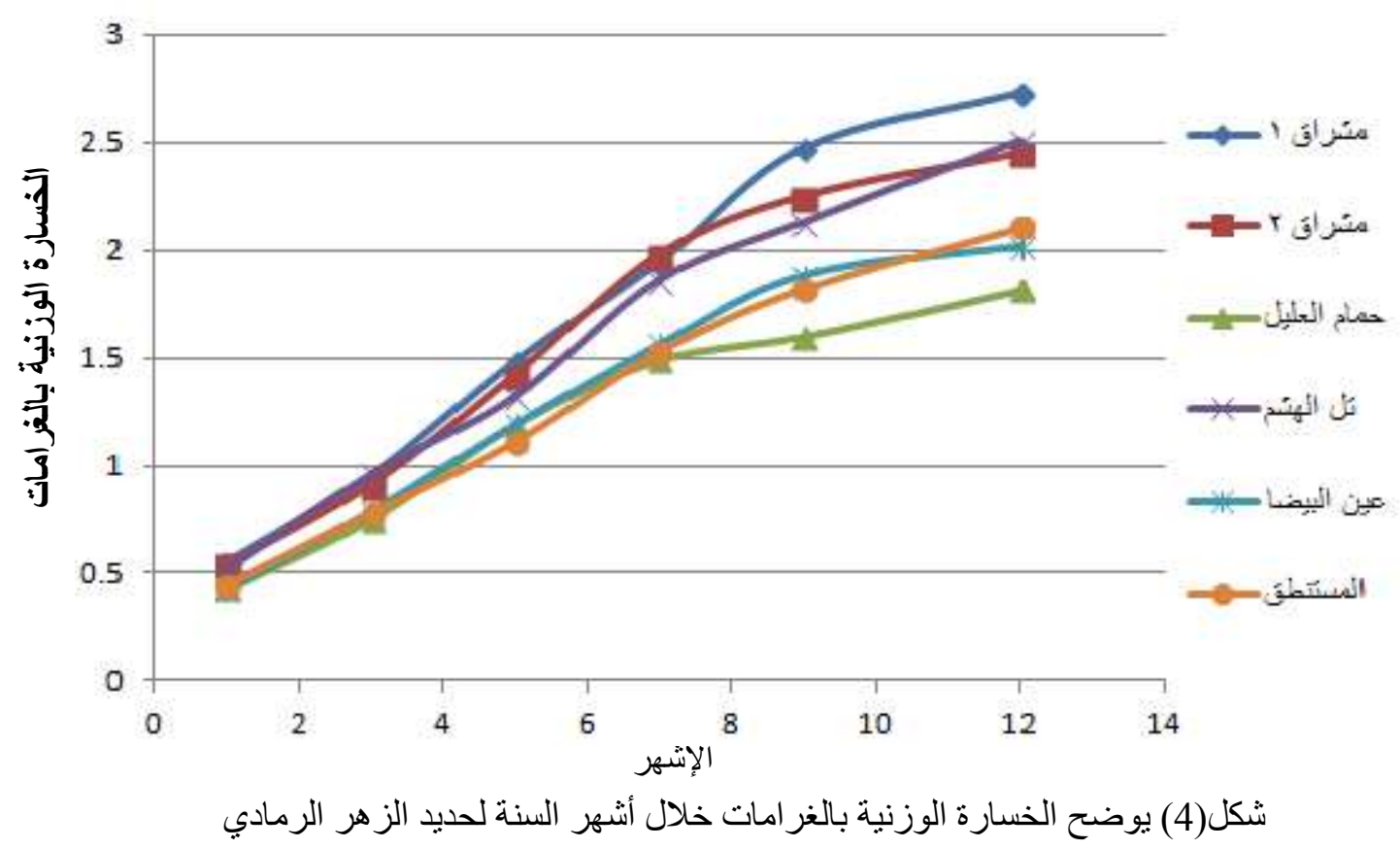

1-6 - 1-6 تصنيف درجات التاكل:

تصنف درجات التاكل (CC) (corrosivity categories) (اعتمادا على معدل اختراق التاكل للسنة الاولى

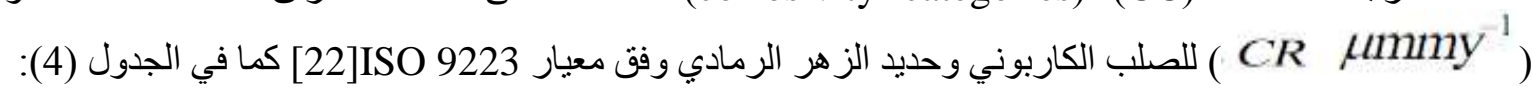
جدول رقم (4) تصنيف درجات التناكل

\begin{tabular}{|c|c|c|c|c|c|}
\hline \multirow{2}{*}{ التصنيف } & الصلب الكربوني & \multirow{2}{*}{$\begin{array}{c}\text { التصنيف } \\
\text { ISO } 9223\end{array}$} & حديد الزهري & \multirow[b]{2}{*}{ المكان } & \multirow[b]{2}{*}{ ت } \\
\hline & $\begin{array}{c}\text { معدل التآكل }{ }^{-1} \text { مRmy } \\
\text { CR }\end{array}$ & & 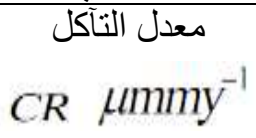 & & \\
\hline $\mathrm{C} 5$ & 384.58 & $\mathrm{C} 5$ & 398.53 & المشر اق (1) & 1 \\
\hline $\mathrm{C} 5$ & 279.27 & $\mathrm{C} 5$ & 327.10 & المشر اق (2) & 2 \\
\hline $\mathrm{C} 4$ & 77.68 & $\mathrm{C} 5$ & 89.32 & حمام العليل & 3 \\
\hline $\mathrm{C} 5$ & 265.23 & $\mathrm{C} 5$ & 279.27 & تل الهشم & 4 \\
\hline $\mathrm{C} 5$ & 89.00 & $\mathrm{C} 5$ & 99.32 & عين البيضا & 5 \\
\hline $\mathrm{C} 5$ & 116.73 & $\mathrm{C} 5$ & 124.29 & المستنطق & 6 \\
\hline
\end{tabular}

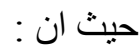

$\mathrm{CR} \mu m m y^{-1}=$ corroision rate micrometer per year.

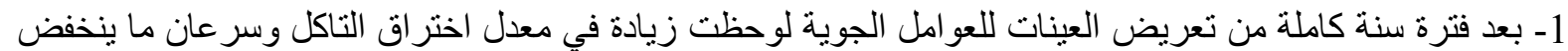

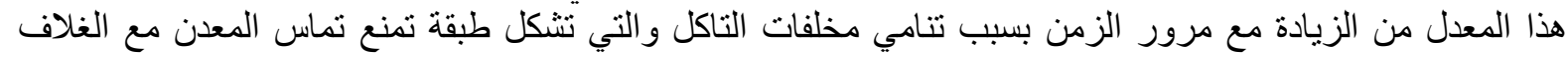

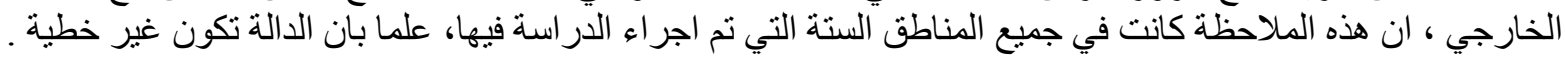


2- كان تاثير SO

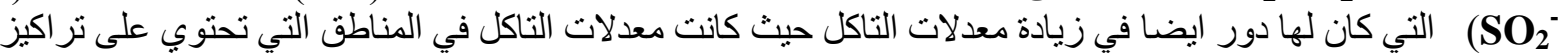
كبيرة من هذه المواد الاعلى وهي كما يلي :المشراق (1) >المشراق(2)> تل الهشتح> المستنطق>عين البيضا>حمام العليل.

3- ابدى حديد الزهر الرمادي تأثرا اكبر بالعو امل الجوية من حيث درجة التنآكل ويعزى ذلك الى الى عاملين هما الاسفنجية

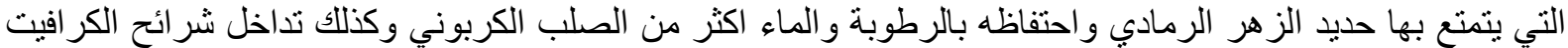
في حديد الزهر و التي تشكل شبكة مستمرة من شقوق الكر افيت التي تسمح بتغلغل وسط التهاء التكل

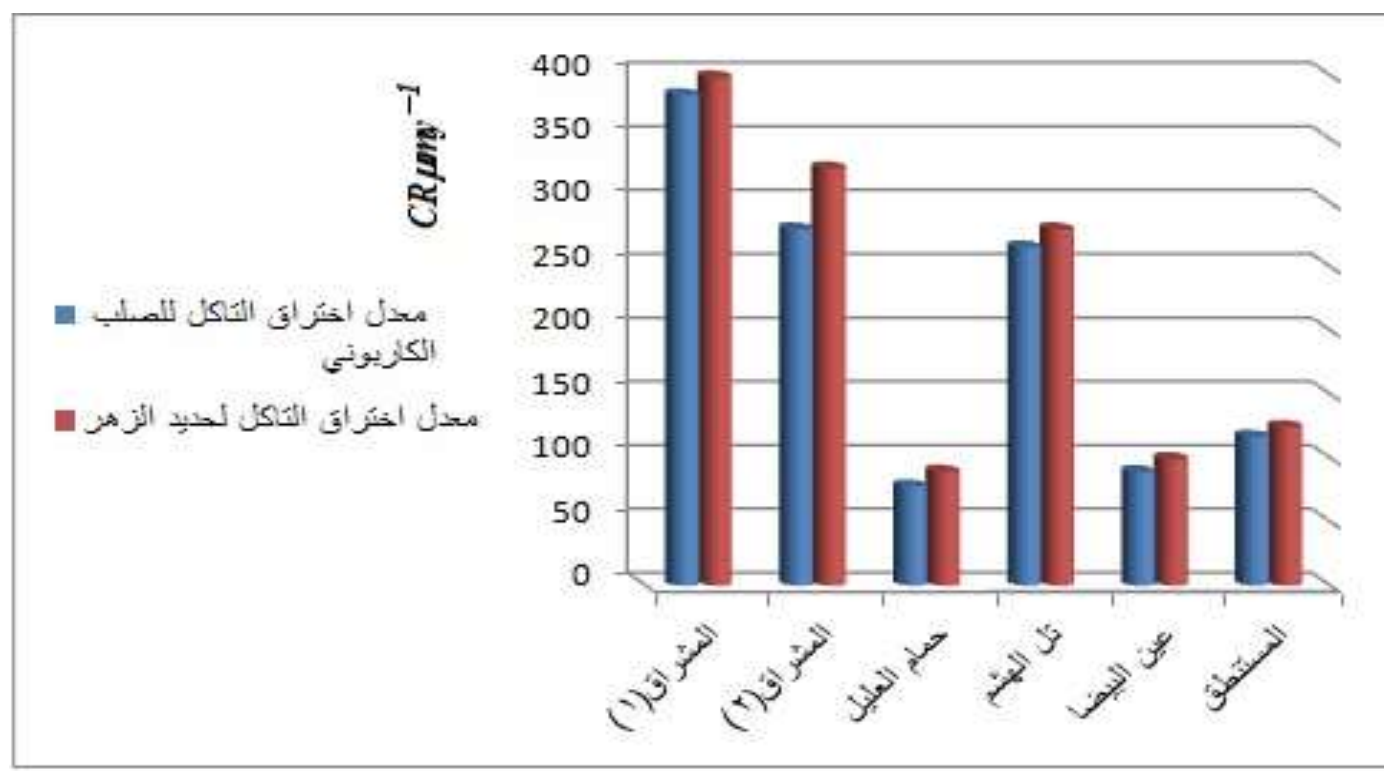

شكل(5) معدل التآكل

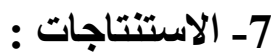
من خلال النتائج السابقة يمكن استتناج ما يلي :

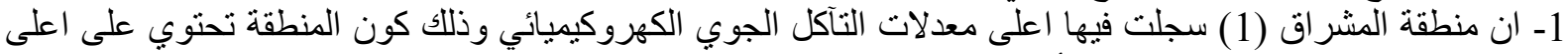

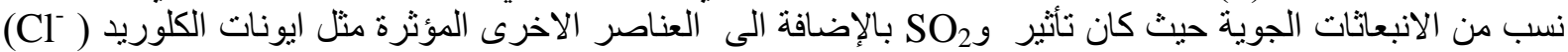
و وايونات الكبريتات ( 2- سجلت منطقة حمام العليل اقل نسبة لدرجة التآكل وذلك كون المنطقة تحتوي على اقل نسب من الانبعانات الجوية حيث كان تأثير و و ( 3ـ كان معدل اختراق التاكل لحديد الزهر الرمادي العات العى من الصلب الكربوني في جميع مناطق البحث

1- J.R. Davis \& Associates, Corrosion understanding the basics ;Asm International ,ISBN:0871706415/563,pp2,2005

2- Ronald .Bianchetti, Peabody's Control Of Pipeline Corrosion ,Nace International The corrosion Society,Houston,Texas,77084,ISBN 1-57590-092-0,2006.

3- Schweitzer, Philip A. Fundamentals of corrosion : mechanisms, causes, and preventative methods , ISBN 978-1-4200-6770-5, CRC Press, Taylor \& Francis Group,pp29,2004. 
4- R. Winston R., Uhlig Corrosion Handbook , John Wiley \& Sons , Inc, 2nd Edition , New York , (2000), PP. 3-343.

5- Peter Brimblecombe, Acid Rain-Deposition to Recovery ,University of East Anglia,Norwich,UK, ISBN;978-1-4020-5884-4(HB)pp31, 2002.

6- Yoon-Seok Choi, Effect of $\mathrm{H} 2 \mathrm{~S}$ on the CO2 corrosion of carbon steel in acidic solutions, Electrochemical Acta 56 (2011) 1752-1760,2005.

$$
\begin{aligned}
& \text { 7- ابر اهيم ،صبحي اسماعيل و عبداله ، ياسر عبدالوهاب، تأثثير نسبة الكربون على مقاومة التآكل للصلب الكربوني }
\end{aligned}
$$

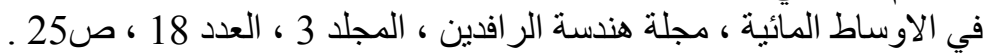

8- Laamari, M.R. et al.," Adsorption and corrosion inhibition of carbon steel in hydrochloric acid medium by hexamethylenediamine tetra(methylene phosphonic acid)". Arabian Journal of Chemistry (2011), doi:10.1016/j.arabjc.2011.03.018.

9- J.L. Rendon And A. Valencia," Kinetics of structural rust transformation in environments containing chloride and $\mathrm{SO}_{2} "$, Madrid Vol Extr, (2003) 9-14.

10- Juan A. Jaen, "Kinetics and structural studies of the atmospheric corrosion of carbon steels in Panama", Hyperfine Interactions 110 (1997) 93-99.

11- Philip.A.Schweitzer, Encyciopedia of Corrosion Technology ,Second Edition,Isbn13:978-0824748784 (March 17;2004),Maryland Edition:2pp3

12- Philip A. Schweitzer, P. E. Corrosion Engineering Handbook Fallston, Maryland, ISBN 0-8247-9709-4 May 22,( 2006), Maryland pp27.

13- Dean.S.W.and Reiser.D.B, Analysis of Long Term Atmospheric corrosion Results from Iso Corrag Program, Outdoor Atmospheric Corrosion ,July 2003.

14- ASTM STP 1421, H. Townsend Ed., American Society for Testing and Materials International, West Conshohocken, PA, 2002.pp5.

15- . Cao X. \& Xu C. C. , "Effect of Chloride on The Atmospheric Corrosion of Simulated Artifact Iron in NO3-Bearing Pollutant Environment " , Acta Metallurgic Sonica English letters, Vol. 19 , NO. 1 ,(2006), PP. 34-42 .

16- Al-Sawaf, F.D. 1977. Sulfate reduction and sulfur deposition in the Lower Fars Formation, Northern Iraq. Economic Geology, 72, 608-618.

17- ISO 8407, Corrosion of metals and alloys - removal of corrosion Products from corrosion test specimens, 1991.

18- Janina M.R. , " Effect of Specimen Preparation on Evaluation of Cast Iron Microstructures " ,Materials Characterization, Vol. 54 , (2005) , PP. 287-304 .

19- ISO 8407, Corrosion of metals and alloys - removal of corrosion Products from corrosion test specimens, 1991.

20- ASTM Designation : G1-90, " Standard Practice for Preparing, Cleaning, and Evaluating corrosion Test Specimens " , 100 Bar Harbor Drive , West Conshohocken , PA 19428-2959, United States , (1999), PP.5 .

21- Cao X. \& Xu C. C. , " Effect of Chloride on The Atmospheric Corrosion of Simulated Artifact Iron in NO3- Bearing Pollutant Environment " , Acta Metallurgical Sinica English Letters , Vol. 19 , NO. 1 ,(2006), PP. 34-42 .

22- ISO 9223:1992 Corrosion of metals and alloys - Corrosivity of atmospheres Classification,2001.

$$
\text { تم اجراء البحث في كلية ألهندسة = جامعة ألموصل }
$$

